\title{
When killers come good
}

DOI:

10.1038/nri1922

URLs

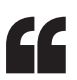

the ability of

NK cells to

recognize and

kill allogeneic

APCs

regulates the

activation of

alloreactive

T cells in skin-

transplantation

5

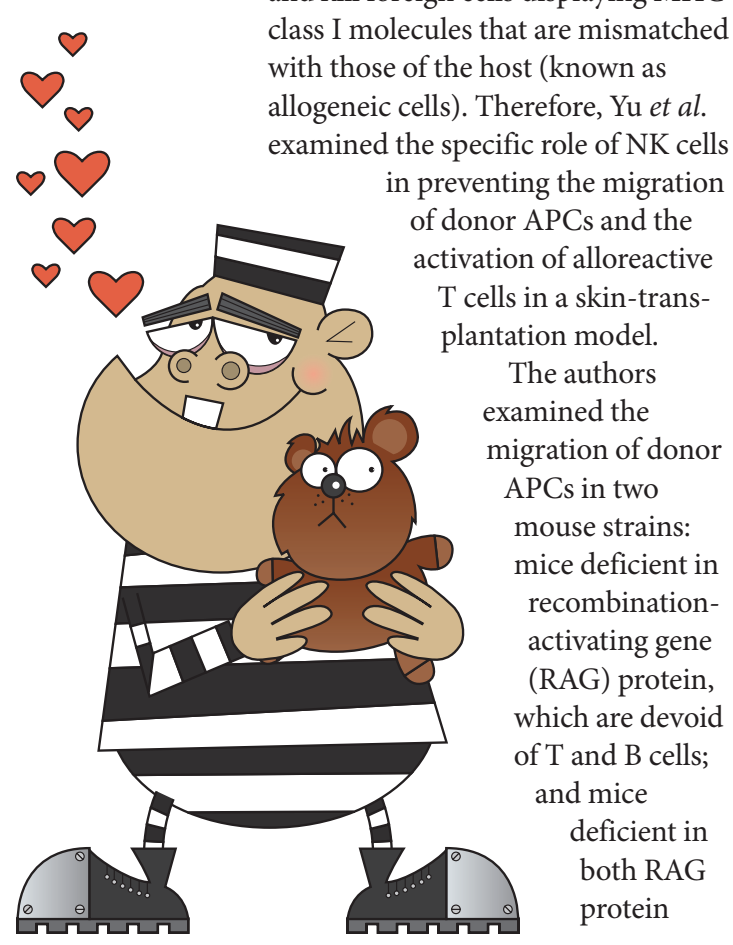

and the common cytokine-receptor $\gamma$-chain $\left(\gamma_{c}\right)$, which additionally lack NK cells. Following transplantation of skin allografts from DBA/2 mice, donor cells (including dendritic cells, DCs) were found in the spleens of RAG- and $\gamma_{c}$-deficient mice but were absent from the spleens of RAG-deficient mice. The authors then directly transferred purified DCs from DBA/2 mice to these two mouse strains. They found high numbers of allogeneic DCs in the spleens, livers and lungs of mice deficient in both proteins but not mice deficient in RAG protein alone, indicating that NK cells have a crucial role in preventing the survival and dissemination of donor DCs in host mice.

To determine whether these allogeneic DCs could stimulate the activation of $\mathrm{T}$ cells in the absence of NK cells, allogeneic DCs from DBA/2 mice were transferred to both strains of mice, and $\mathrm{T}$ cells that were genetically similar to those of the host mice were transferred 2 weeks later. Transferred T cells that were recovered 3 days later from the spleen, liver and lungs of RAG- and $\gamma_{c}$-deficient mice had undergone multiple rounds of cell division and readily produced interferon- $\gamma$, whereas T cells that were recovered from RAG-deficient mice had not undergone cell division. These data indicate that, in the absence of NK cells, allogeneic DCs can induce the activation of alloreactive $T$ cells at multiple sites in host mice.

So the ability of NK cells to recognize and kill allogeneic APCs regulates the activation of alloreactive $\mathrm{T}$ cells in skin-transplantation models, highlighting that NK cells are potential therapeutic targets for the induction of tolerance to transplants.
ORIGINAL RESEARCH PAPER Yu, G. et al. NK cells promote transplant tolerance by killing donor antigen-presenting cells. J. Exp. Med. 24 July 2006 (doi:10.1084/jem.20060603) 